\title{
Microwave characterization of bio-composites materials based finite element and Nicholson-Ross-Weir methods
}

\begin{abstract}
In this work, Bio-composite of oil palm empty fruit bunch fibre (OPEFB)-filler and polycaprolactone (PCL)-polymer has been prepared and characterized. The functional groups and morphology of the prepared samples were characterized by Fourier transform infrared spectroscopy (FT-IR). By using the Nicholson- Ross-Weir (NRW) mode, both of real and imaginary relative permittivity values of the samples were obtained simultaneously from the reflection and transmission coefficient measurements of the materials. Whereas, the attenuation with the field distribution at the waveguide filled with a sample were considered by using the Finite Element Method (FEM). The magnitude of the reflection and transmission $(\mathrm{R} / \mathrm{T})$ coefficients of the composite with different filler percentages were measured using rectangular waveguide in conjunction with a microwave vector network analyzer (VNA) in $\mathrm{X}$-band range of frequency. The computations of the S-parameters were achieved by using the FEM technique along with NRW mode. Then, the obtained results were compared with the measured R/T coefficients. Relative error results nominated the FEM mode due to its highly accurate results than the other method.
\end{abstract}

Keyword: Microwave measurements; OPEFB; PCL; Bio-composite 